\title{
La Bretagne, une illusio qui fonctionne
}

Brittany: an illusio that works

Laurent Le Gall

\section{Q OpenEdition}

Journals

Édition électronique

URL : https://journals.openedition.org//bl/1152

DOI : $10.4000 / \mathrm{lbl} .1152$

ISSN : 2727-9383

\section{Éditeur}

Université de Bretagne Occidentale - UBO

\section{Édition imprimée}

Date de publication : 1 mars 2014

Pagination : 111-136

ISBN : 979-10-92331-07-3

ISSN : 1270-2412

\section{Référence électronique}

Laurent Le Gall, « La Bretagne, une illusio qui fonctionne », La Bretagne Linguistique [En ligne], 18 | 2014, mis en ligne le 01 mai 2021, consulté le 21 mai 2021. URL : http://journals.openedition.org/lbl/1152 ; DOI : https://doi.org/10.4000/lbl.1152

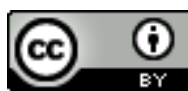

La Bretagne Linguistique est mise à disposition selon les termes de la Licence Creative Commons Attribution 4.0 International. 


\section{La Bretagne, une illusio qui fonctionne ${ }^{1}$}

\section{$\mathrm{P}$}

arlons d'un objet qui peut fâcher en essayant de le traiter avec les outils des sciences sociales quand bien même nous ne sommes pas dupe de la caractérisation idéologique qui peut sous-tendre tel ou tel pan de l'ordre du discours scientifique. Comme il y a un discours régionaliste de l'identité, il y a un discours républicain de l'identité. Les deux convoquent quelquefois les mânes de l'opération intellectuelle pour exprimer ce qu'ils ont foncièrement à démontrer. Gageons que ce travail, critiquable par essence, ne pâtira pas de cette seule critique. Nous nous serons sinon trompé de but.

Le titre de cette contribution, «La Bretagne, une illusio qui fonctionne», mérite quelques explications liminaires. Il fait appel au concept d'illusio dont Pierre Bourdieu donne la définition suivante :

«En fait, le mot intérêt, dans un premier sens, voulait signifier très précisément ce que j'ai mis sous cette notion d'illusio, c'est-à-dire le fait d'accorder à un jeu social qu'il est important, que ce qui s'y passe importe à ceux qui y sont engagés, qui en

* Maître de conférences d'histoire contemporaine, CRBC (EA 4451-UMS 3554), $\mathrm{UBO} / \mathrm{ueb}$.

1. Cet article a paru sous une forme amplifiée : Jean-François Simon et Laurent LE GALL, «La Bretagne par intérêt», Ethnologie française, vol. 42, n 4, 2012, p. 771-886. 
sont. (...) L'illusio, c'est donc le contraire de l'ataraxie, c'est le fait d'être investi, d'investir dans des enjeux qui existent dans un certain jeu, par l'effet de la concurrence, et qui n'existent que pour les gens qui, étant pris dans ce jeu et ayant des dispositions à reconnaître les enjeux qui s'y jouent, sont prêts à mourir pour des enjeux qui, à l'inverse, apparaissent comme dépourvus d'intérêt du point de vue de celui qui n'est pas pris à ce jeu, et le laissent indifférent ${ }^{2}$.»

En appliquant cette définition à la manière dont se construit le marché identitaire en Bretagne, l'on voudra peut-être bien constater que l'identité régionale est le produit d'enjeux sous-jacents que le sens commun dont elle est nimbée (ouverture, métissage, apaisement) dans les discours de certains de ses porte-parole ne laisse pas percevoir d'emblée.

Il s'agira donc ici de cheminer à l'intérieur de ces discours qui émanent de très nombreux agents, d'essayer de déterminer comment l'identité en tant que lieu commun sécrète sans cesse de l'identification et comment le Conseil régional de Bretagne, à travers sa politique culturelle en particulier, s'en fait aussi, sous couvert d'une neutralisation des enjeux, l'un des grands sinon le grand orchestrateur.

\section{Identification}

L'identité bretonne serait donc un fait. À tout le moins pour ceux qui, décennie après décennie, en ont fait la pierre de touche de leur combat politique au nom d'une rhétorique de la spécificité régionale. De mieux en mieux connue même si elle se perd quelquefois dans le dédale des mouvements qui ont essayé de prospérer sur des particularités érigées en particularismes ${ }^{3}$, l'histoire de la mouvance régionaliste - au sens large - a été marquée par l'échec d'un projet qui, quel que fût son but (autonomie pour l'Union démocratique bretonne, indépendance pour Emgann), ne rencontra et ne rencontre toujours qu'un très faible écho dans la société.

2. Pierre Bourdieu, Raisons pratiques. Sur la théorie de l'action, Paris, Minuit, 1984, p. 151-152.

3. Cf. Yann Fournis, Les régionalismes en Bretagne. La région et l'État (19502000), Bruxelles, Peter Lang, 2006. 
Il ne s'agit pas de revenir ici sur les initiatives et les métamorphoses de cette galaxie demeurée marginale mais de constater, à ce stade, et sans toutefois vouloir relier mécaniquement les phénomènes, que «la construction d'un paysage identitaire ${ }^{4} »$ fut d'autant plus réalisable que de nombreux intellectuels y ont contribué peu ou prou. En participant, à travers leurs travaux, à l'élaboration d'un sens commun associant étroitement Bretagne et identité, des universitaires, et non des moindres au regard de leur position dans le champ académique, n'ont cessé de produire un discours autorisé sur la singularité péninsulaire. Nous prendrons trois exemples parmi ces figures marquantes. Reconnu pour l'impulsion qu'il a apportée à l'avènement d'une histoire culturelle de la France moderne, Alain Croix a signé, il y a quelque temps, un ouvrage grand public intitulé La Bretagne entre histoire et identité dont les dernières lignes décrètent : "Dans la Bretagne du début du XXI siècle, il n'est plus nécessaire de choisir entre l'ouverture et l'identité : si l'image n'était politiquement connotée, on pourrait retenir que la "force tranquille" de cette identité ne garantit évidemment rien pour l'avenir, mais qu'elle permet tout ${ }^{5} »$. Appelé à préfacer un manuel d'histoire de la Bretagne destiné aux collégiens et patronné par le Centre régional de documentation pédagogique, Yves Le Gallo, professeur de civilisation de la Bretagne à l'Université de Brest et père fondateur du Centre de recherche bretonne et celtique, titra sa contribution : «Bretagne, le sursaut d'un destin manqué ${ }^{6} »$. L'on pariera volontiers que la Composition française de Mona Ozouf a dû en partie son succès à deux raisons au moins : l'indéniable qualité littéraire de la mise en récit d' ' une enfance bretonne»; la critique, majoritairement admise de nos jours, d'un ordre républicain qui, pour n'avoir «pu se défaire de son surmoi jacobin ${ }^{7} »$, aurait nivelé et uniformisé au

4. Barbara LOYER, «Bretagne», dans Béatrice Giblin (dir.), Nouvelle géopolitique des régions françaises, Paris, Fayard, 2005, p. 277-325, p. 302.

5. Alain CroiX, La Bretagne entre histoire et identité, Paris, Gallimard, 2008, p. 127.

6. Yves Le Gallo, «Bretagne, le sursaut d'un destin manqué », dans Louis Élégoët, Bretagne, une histoire, Rennes, Centre régional de documentation pédagogique, 1998, p. 5-8, p. 5.

7. Mona Ozouf, Composition française. Retour sur une enfance bretonne, Paris, Gallimard, 2009, p. 228. 
prétexte qu'il incarnait un Universel et la Bretagne, pour toutes sortes de raisons (de la langue à l'assignation chouanne), son antonyme. Que ces discours qui systématisent l'identité en empruntant des points de vue différents ressortissent à des itinéraires intellectuels contrastés, cela est évident : passage d'un militantisme politique au Parti communiste français à un militantisme éditorial au cœur duquel se situe quasi exclusivement la Bretagne pour Alain Croix $^{8}$; défense d'un pré carré institutionnel pour un acteur de la grande transformation finistérienne de l'après-guerre chez un Yves Le Gallo dont la carrière fut marquée par une confession braudelienne d'aprèsagrégation, vécue comme une vexation, et l'exigence d'un bond en avant bas-breton qui se devait d'être un impératif ${ }^{9}$; ombre portée du père absent, Yann Sohier, instituteur, militant breton et fondateur d' $\mathrm{Ar}$ Falz, sur la trajectoire de la philosophe qui deviendra par la suite, en compagnie de François Furet, la dépositaire d'un nouvelle histoire de la Révolution française débarrassée abruptement de ses oripeaux marxistes. Que ces discours se réclament d'une démarche commune aux travaux qui s'ingénient à cerner les processus d'invention des entités sociales et territoriales, rien de surprenant non plus dans la mesure où ils se sont intégrés à la vague constructiviste qui a dominé jusqu'à récemment l'historiographie contemporaine des identités ${ }^{10}$. Qu'ils soient, enfin, symptomatiques du couronnement d'un «roman régional»-comme il a existé un «roman national ${ }^{11} »$ - nourri de ses héros (Anne de Bretagne), de ses temps forts (l'État ducal des XIV

8. Alain Croix, «Marx, la chaisière et le petit vélo», dans Jean-Pierre Rioux et Jean-François Sirinelli (dir.), Pour une histoire culturelle, Paris, Le Seuil, 1997, p. 51-71.

9. Yves Le GALlo, «Souvenirs d'un universitaire bas-breton», dans Études sur la Bretagne et les pays celtiques. Mélanges offerts à Yves Le Gallo, Brest, Centre de recherche bretonne et celtique, 1987, p. 7-17, p. 14-17.

10. Cf. Martina Avanza et Gilles Laferté, «Dépasser la "construction des identités"? Identification, image sociale, appartenance», Genèses, ${ }^{\circ} 61$, 2005, p. 134-152 ; Blaise WiLfERT-PorTAL, «Nation et nationalisme», dans Christian Delacroix, François Dosse, Patrick Garcia et Nicolas Offenstadt (dir.), Historiographies, tome 2 : Concepts et débats, Paris, Gallimard 2010, p. 10901102.

11. Anne-Marie Thiesse, La création des identités nationales. Europe, XVIIIXXe siècle, Paris, Gallimard, 2001 [1999], p. 133 ; Pierre NorA, Présent, nation, mémoire, Paris, Gallimard, 2012, p. 7-29. 
et $\mathrm{XV}$ e siècles, l'«âge d'or» de la Bretagne des enclos paroissiaux) et de ses blessures (Conlie, la guerre de 1914-1918 et leurs hécatombes, pour ne citer que des nœuds mémoriels gorgés encore quelquefois d'affects), et voilà qui ne cesse d'alimenter le questionnement sur les relations entre sa fabrication recommencée, son utilité, son utilisation et la demande sociale, avérée ou postulée, à laquelle il serait censé répondre.

Adoubé par des travaux académiques qui, tout en soulignant les pièges de l'essentialisation, participent quelquefois à l'authentification d'un continuum historique cimentant, "grâce» à l'opération historienne, une société perçue dans son unicité ${ }^{12}$, le lexique de l'identité est devenu, au fil du temps, une valeur sinon refuge tout au moins partagée sans la moindre équivoque par la très grande majorité des acteurs du champ intellectuel et politique régional. C'est peut-être là une des forces de ce lexique dont Rogers Brubaker a souligné combien sa fonction de catégorie d'analyse opacifiait considérablement «la double orientation de beaucoup d'identitairiens académiques qui sont à la fois des analystes et des protagonistes des politiques identitaires $\left.{ }^{13}{ }^{\prime}\right)$. Entourée fréquemment de précautions oratoires et certifiée en tant qu'objet scientifique légitime par une génération d'universitaires soucieux de ne pas être taxés de «dérive identitaire», soumise à la question, cette identité-qui-neva-pas-de-soi porte en elle, parce qu'elle ne va pas ou plus de soi justement - sauf dans certains milieux indépendantistes qui n'ont pas compris les dividendes que permettait une démarche constructiviste parée des bienfaits de la désidéologisation apparente -, toutes sortes d'investissements qui vont du diagnostic à la mise en évidence d'un substrat commun ou d'un modèle particulier. Dénominateur

12. Joël Connette, Histoire de la Bretagne et des Bretons, tome 1 : Des âges obscurs au règne de Louis XIV, tome 2 : Des Lumières au XXIe siècle, Paris, Le Seuil, 2008 [2005]; la première phrase de la quatrième de couverture souligne : «Devenue française en 1532, la Bretagne n'a jamais cessé d'être elle-même». Dans son introduction, Joël Cornette rappelle par exemple : «La "région" Bretagne se distingue d'abord par cette résistance à la sujétion, une soif de reconnaissance, une fièvre identitaire qui semble s'opposer à toute entreprise d'unification, d'amalgame dans un moule commun", p. 11.

13. Rogers BRUBAKER, «Au-delà de l"“identité "», Actes de la recherche en sciences sociales, $\mathrm{n}^{\circ} 139,2001$, p. 66-85, p. 70. 
couramment admis à tel point que sa présence - qui n'est autre que la sanction de sa naturalisation - imprègne un tissu culturel qu'irriguent aussi bien la presse locale que des ouvrages savants, l'identité bretonne, même lorsqu'elle est pensée sous l'aspect d'une identité-problème ou, plus exactement, puisqu'elle est pensée de la sorte, sécrète, ce faisant, de l'identification.

Parce qu'elle est devenue un lieu commun, l'identité suppose qu'elle soit auscultée toujours davantage par ceux qui y ont un intérêt aux fins d'une (ré)assurance sur sa consistance et/ou d'une meilleure compréhension de sa genèse et de ses dynamiques - c'est selon. Nombreux sont ainsi les acteurs en quête de cette certification qui repose sur son actualisation. Nous en voudrons pour preuve les multiples sources d'information émanant d'institutions ou d'associations qui, pour des raisons différentes, s'y engagent plus ou moins pleinement : le Comité régional du tourisme de Bretagne, le think tank Bretagne Prospective, le mensuel Bretons (créé en 2005 et émanation de Ouest-France) ou encore ArMen, revue dédiée à «La Bretagne, un monde à découvrir», pour ne citer qu'eux, participent à la concrétisation de ce qu'est et doit être la, ou plutôt, leur région. Penchons-nous succinctement sur ArMen. Devenue son fonds de commerce en se démarquant progressivement d'un projet initial bâti, dès 1986, sur une connaissance anthropologique de la péninsule, l'identité telle qu'elle s'écrit actuellement dans ses colonnes apparaît davantage comme une entreprise de classement de ses attributs d'où la performativité est loin d'être exclue voire même assumée - sur le mode : dire la Bretagne, c'est la faire. Un changement de registre qui serait supposément en adéquation avec l'air du temps ${ }^{14}$.

Ce discours identificatoire qui procède d'un travail de sélection des données, c'est-à-dire d'une opération visant à en distinguer certaines plutôt que d'autres, relève de ce que nous qualifierons arbitrairement de double logique, l'une ordinaire (usuelle si l'on préfère) et l'autre savante. Il repose sur la capacité qu'ont des acteurs, en fonction de leur position et de leurs compétences, à abonder la notion d'identité en topiques renouvelés qui peuvent contribuer, en fonction

14. Cf. Charlotte Colliou, ArMen et la création d'une identité bretonne (19862010), mémoire de master 2 (Yvon Tranvouez, dir.), Brest, Université de Bretagne Occidentale, 2010. 
de la réception qui leur est faite, à en élargir la base. Deux objets que nous verserons dans la première catégorie (ordinaire) retiendront ici notre attention. Le premier est ténu. À défaut d'être une idée neuve, le bonheur serait, si l'on en croit certains observateurs/prescripteurs, une idée bretonne. Il n'entre pas dans notre propos de retracer les voies et les raisons de sa réification - à rebours des flux migratoires qui ont marqué la démographie bretonne jusque dans les années 1960, l'attractivité des quatre départements, que signale l'installation annuelle de 25000 nouveaux arrivants, en est une -, mais de constater son inscription définitive dans le grand inventaire de la spécificité régionale. Au plus fort de la saison touristique, Le Télégramme a publié, en août 2011, une enquête en six volets. Son titre : "Le bonheur de vivre en Bretagne». Convoqués au ban de l'analyse, «le modèle breton» défini par Hubert Coudurier, le directeur de la rédaction, et ses déclinaisons, qu'il s'agisse de la "qualité de vie enviée» (9 août) ou de «l'identité bretonne, un moteur social» (13 août). Rien que de très «naturel» pour un journal justifiant son existence par son localisme et tirant parti, à l'instar de son concurrent Ouest-France, des bénéfices inhérents à son expression la plus diserte ${ }^{15}$. Et, partant, rien que de très normal à ce que la rhétorique d'une «identité bretonne (...) parfaitement palpable» et qui, « depuis quelques décennies (...) se nourrit du quotidien» (13 août), coïncide avec la valorisation d'un bonheur délimité et défini à l'ombre de frontières régionales. En postfaçant la thèse de Ronan Le Coadic dévolue à L'Identité bretonne, Michel Wieviorka ne disait pas autre chose à l'extrême fin du vingtième siècle. Clin d'œil à l'ouvrage de Morvan Lebesque qui avait connu un certain retentissement au tout début des années $1970^{16}$, son intitulé, «Comment peut-on ne pas être Breton?», renvoyait à la lecture élogieuse de l'analyse et formalisait un peu plus encore, avec l'onction du sociologue, l'existence d'une équation alliant Bretagne et positivité :

«Dans un monde qui se globalise économiquement, et se fragmente culturellement, elle [l'identité bretonne] offre un

15. Thibault COURCELle, «Le rôle de la presse quotidienne régionale bretonne dans la création d'une "identité bretonne" : étude comparative de Ouest-France et du Télégramme», Hérodote, $\mathrm{n}^{\circ}$ 110, 2003, p. 129-148.

16. Morvan LeBesque, Comment peut-on être Breton? Essai sur la démocratie française, Paris, Le Seuil, 1970. 
exemple, certes fragile et limité, d'articulation d'une affirmation culturelle vivante et en devenir, d'une capacité d'action économique et d'une ouverture politique profondément démocratique : comment, dès lors, ne pas se sentir Breton ${ }^{17}$ ?»

Le second objet concerne la fête. Combien d'articles, de papiers, de supports qui ne louent la vitalité festive d'une Bretagne dont elle serait, à en croire ses thuriféraires, une de ses expressions les plus achevées. Fruit de la collaboration entre Historia et Ouest-France, un hors-série récent s'est penché sur une fête plurielle et efflorescente entendue, à travers la plupart des contributions, comme la matrice d'un «vouloir vivre ensemble irrépressible» attribué à la population et gagé sur une "une tradition "d'être ensemble" ${ }^{18}$ ». Organisée par le Conseil régional, la Breizh Touch*19, qui mit «Paris à l'heure bretonne» du 20 au 23 septembre 2007 et vit défiler 3000 danseurs et musiciens des cercles et bagadoù sur les Champs-Élysées, devait consacrer l'image flatteuse de cette péninsule à la mode (et non à la mode de Bretagne) dont la communication officielle de la collectivité territoriale traça les contours en ces termes : «Elle est belle, intelligente, cultivée, entreprenante... Non, il ne s'agit ni d'une comédienne à succès, ni d'un top-model en vogue mais d'une région. Et pas n'importe laquelle : la nôtre ${ }^{20} \gg$. Affecté à la promotion de la vie culturelle, le site internet Vibrez, qui dépend du site du Comité régional du tourisme financé à $80 \%$ par la Région ${ }^{21}$, consacre une page à la Fête de la Bretagne. Le visiteur peut y lire :

«Cerise sur le kouign aman, phare breton éclairant le temps d'une éphéméride l'ensemble de la Bretagne, la Saint-Yves est à la région ce que les férias sont au sud-ouest de la France. L'occasion pour tout un peuple de se souvenir de ses origines pour mieux construire son avenir. Générosité, hospitalité, solidarité, justice. Des valeurs cultivées à la mode de chez eux par les

17. Michel WieviorkA, «Comment peut-on ne pas être Breton?», dans Ronan Le Coadic, L'Identité bretonne, Rennes, Terre de Brume-Presses Universitaires de Rennes, 1998, p. 429-432, p. 432.

18. Historia, 2011, p. 3 et 7.

19. Sur les supports de communication, l'astérisque renvoie à la traduction «Esprit Bretagne» pour Breizh Touch*.

20. Bretagne ensemble-Breizh a gevret, $\mathrm{n}^{\circ}$ 6, 2007, p. 24.

21. La Région avec un $\mathrm{r}$ majuscule renvoie à la collectivité territoriale. 
Bretons depuis le quatorzième siècle et la canonisation de Yves Hélory de Kermartin. Une devise immuable, à laquelle les fondateurs de la Fest-Yves ont juste ajouté la festivité. Chaque année, au mois de mai, cette déclaration " univercelte " est adressée aux Bretons de Bretagne, mais aussi à la nombreuse diaspora faisant flotter le Gwenn ha Du sur tous les toits du monde ${ }^{22}$.»

On l'aura compris, la Bretagne serait aussi une fête. Constat historique à forte teneur prédictive, matérialisation symbolique sous la forme d'un grand raout parisien, publicité saturée de "bons mots» et de clichés à destination des consommateurs de la société des loisirs : la convergence des registres qui reposent tous sur un encodage identitaire d'un fait pourtant universel renvoie à ce processus de régionalisation d'une culture ${ }^{23}$ dont les promoteurs garantissent l'authenticité pour s'être montrés capables - leur statut leur tient lieu de justification - d'en authentifier les empreintes. La fête tombe ici à point nommé : se prêtant à la fois aisément à une valorisation des (ré)inventions successives de la tradition et à la consécration de son contenu métissé, autant de ressources passives aisément mobilisables pour réfuter les imputations d'un repli sur soi, elle avait tout pour devenir ce parangon d'une identité ouverte, en prise avec le monde, à condition qu'elle puisse être identifiée - et, donc, inventée - de la sorte. Ce à quoi, par transferts réciproques ou appropriations mimétiques de quelques mêmes thématiques, des structures aussi différentes que celles que nous avons mentionnées plus haut se sont employées pour en faire un marqueur régional spécifique qui semble désormais aller de soi. Ainsi en va-t-il de l'identification puis de la routinisation de ces nouveaux points d'appui identitaires par absolutisation progressive de ce qui n'était, jadis ou naguère, qu'à l'état de formes matérielles ou de traits culturels : un double processus qui, parce qu'il paraît bénéficier d'un très large consentement social - quels peuvent être, en l'occurrence, les détracteurs de la fête et du bonheur ? -, ne cesse d'accréditer l'idée selon laquelle l'effectivité de l'identité serait bien

22. https://web.archive.org/web/20120331190636/http://vibrez.tourismebretagne. com/la-bretagne-festive/fete-de-la-bretagne (16 février 2012).

23. Georges RAVIS-GIORDANI, «La Corse : culture régionale? culture régionalisée ?», Ethnologie française, vol. 33, n 3, 2003, p. 451-458, p. 451. 
réelle puisqu'elle ne cesserait de se régénérer par ajouts successifs d'objets potentiellement et aisément labellisables.

L'identification repose aussi sur une logique savante. La «bretonnité», pour reprendre un terme couramment admis chez ceux qui l'étudient et, quelquefois, la fabriquent, ne serait-ce que nominalement, a ses spécialistes attitrés à l'Université. Elle est, in fine, au cœur d'un travail de qualification qui applique à des pans entiers de la réalité sociale observée la notion de modèle. À la suite du géographe Corentin Canévet qui préconisa, dans les années 1970, l'utilisation de l'expression "modèle agricole breton» - ce sera le titre de l'ouvrage tiré de sa thèse d'État ${ }^{24}$ - pour expliquer la grande métamorphose des campagnes de l'Ouest après la Seconde Guerre mondiale, se sont multipliées les déclinaisons d'une expression que garantissait de son autorité scientifique un livre ambitieux et remarqué. «Modèle industriel breton» sous la plume du géographe Michel Phlipponneau, membre actif du Comité d'étude et de liaison des intérêts bretons (Célib), dont il fut le vice-président de 1961 à 1967, et défenseur de la cause régionale au nom de laquelle il écrivit Debout Bretagne! en $1970^{25}$, «modèle électoral breton» révélé tout au long de la très minutieuse étude menée sur le comportement électoral de ses contemporains par l'historien Jean-Jacques Monnier, militant de 1'Union démocratique bretonne par ailleurs ${ }^{26}$, «modèle identitaire breton» soumis au point d'interrogation avant d'être approuvé tel quel dans la lecture préfacière que Michel Denis, historien, ancien président de l'Université Rennes 2 et soutien mesuré de certaines aspirations locales ${ }^{27}$, proposa de l'ouvrage de Ronan Le Coadic ${ }^{28}$ :

24. Corentin CANÉVET, Le modèle agricole breton. Histoire et géographie d'une révolution agro-alimentaire, Rennes, Presses Universitaires de Rennes, 1992.

25. Michel Phlipponneau, Le modèle industriel breton, 1950-2000, Rennes, Presses Universitaires de Rennes, 1993.

26. Jean-Jacques MonNier, Le comportement politique des Bretons, 1945-1994, Rennes, Presses Universitaires de Rennes, 1994.

27. Cf. l'hommage collectif qui lui a été rendu sous le titre «Michel Denis (19312007), un historien-citoyen », dans l'ouvrage de Claude GESLIN, Patrick GouRLAY, Jean-Jacques MonNIER, Ronan Le COADIC et Michel Denis, Histoire d'un siècle. Bretagne 1901-2000. L'émancipation d'un monde, Morlaix, Éditions Skol Vreizh, 2010, p. 11-13.

28. Michel Denis, «Préface», dans Ronan Le Coadic, L'Identité bretonne, op. cit., p. $9-11$, p. 10 . 
le succès de la formule qui, à bien des égards, introduit un risque tautologique dans le découpage des objets et les argumentaires qui en découlent - toute recherche produite sur la Bretagne ne pourrait déboucher consubstantiellement que sur un modèle breton... - en dit long sur une typification de la région parée de ces critères scientifiques qui, en la légitimant, contribuent à imposer sa normativité. On ne s'étonnera peut-être pas alors que l'entretien donné au magazine de sa collectivité par le président socialiste du Conseil régional, l'agrégé d'histoire Jean-Yves Le Drian, dans la foulée de sa réélection du printemps 2010, eut pour titre : «Définir ensemble un nouveau modèle breton ${ }^{29} \gg$. Et l'on notera incidemment qu'à l'image de l'identité, ce mot fréquemment valise même lorsqu'il sert de catégorie d'analyse ${ }^{30}$, le terme de modèle offre aussi à chacun la possibilité de puiser dans sa double acception de paradigme et d'exemple à suivre (voire auquel se conformer).

Résumons-nous. Produit plus ou moins conscientisé d'une constellation d'observateurs/prescripteurs de l'identité bretonne, la standardisation de cette notion, qui repose sur la conjonction de discours convergents émanant d'agents différemment positionnés dans l'espace social et dans le temps, semble constituer aujourd'hui un lieu à ce point commun que toute critique peut passer pour une atteinte à l'existence d'un ordre régional fondé, entre autres, sur «l'affirmation d'un maillage de relations logiques entre le concept de région et un ensemble d'éléments qui la définiraient tout entière ${ }^{31} »$. «La bataille de l'opinion est gagnée», affirmait au Télégramme, dans l'enquête sus-mentionnée, un «journaliste et producteur TV (...) siége[ant] depuis deux ans au Conseil culturel de Bretagne» (13 août) : consensuelle en apparence, quand bien même une étude socio-historique des enjeux contradictoires liés à son objectivation, son instrumentalisation et aux ressorts de son intimisation nuancerait vraisemblablement des idées reçues et attesterait combien les années 1990 ont constitué un tournant dans sa mise en forme, l'identité serait devenue ce ciment collectif au nom duquel il serait loisible de penser et le passé, et le futur. Ce dont d'ailleurs ne se privent

29. Bretagne ensemble-Breizh a gevret, $\mathrm{n}^{\circ} 14,2010$, p. 4.

30. Cf. Rogers BrubaKer, «Au-delà de 1'“ identité "», op. cit.

31. Barbara LOYER, «Bretagne», op. cit., p. 305. 
guère certains de ses experts qui, parce qu'experts conçoivent qui les «scénarios d'un passage à l'acte [décentralisateur ? autonomiste ? indépendantiste ? ${ }^{32} »$, appellent qui des mesures concrètes pour pallier ce «sentiment d'indétermination, cette difficulté d'appréhender l'existence et le réel» attribués à des Bretons «privés d'un minimum d'éducation et d'enseignement sur leur lieu de vie et d'habitation, sur leur histoire, leur identité ${ }^{33} \gg$. Autant de ramifications d'un marcottage identitaire qui ne dit pas son nom et dont il convient d'apprécier, autant que faire se peut, l'intérêt qui lui est conféré à l'intérieur du champ politique borné par la Région.

\section{Neutralisation?}

On l'aura peut-être remarqué : le politique court en filigrane dans cette contribution. Et pour cause : en soulignant, il y a vingt-cinq ans déjà, combien «la définition d'une "culture nationale" fait partie du travail politique d'“authentification" de l'identité collective», Louis Pinto invitait à voir dans la nation une "fiction politique» dont le succès dépendait, entre autres, des moyens alloués par ceux qui entendaient être ses porte-parole ${ }^{34}$. Rapportée à la Bretagne contemporaine, cette proposition mérite que l'on s'y intéresse de près afin d'en éprouver ou non la pertinence. Commençons par des choses connues et qui ne prêtent plus guère à discussion. En dépit de sa longue tradition, le mouvement politique régionaliste a failli dans la conquête des suffrages et l'imposition de ses projets, facilitant ainsi, à compter des années post-soixante-huitardes, l'émergence puis la consolidation de revendications à teneur identitaire portées presque exclusivement par les seuls opérateurs culturels ${ }^{35}$. En coïncidant avec l'éclatement progressif du «modèle politique français» marqué par

32. Ronan LE COADIC, Bretagne, le fruit défendu ?, Rennes, Presses Universitaires de Rennes, 2002, p. 160-163.

33. Jean Ollivro, Les paradoxes de la Bretagne. Quelques clés pour le développement de la Bretagne, Rennes, Apogée, 2005, p. 39.

34. Louis PINTO, «Une fiction politique : la nation. À propos des travaux de Jenö Szücs», Actes de la recherche en sciences sociales, $n^{\circ} 64,1986$, p. 45-50, p. 50.

35. Cf. Yann Fournis, Les régionalismes en Bretagne. La région et l'État (19502000), op. cit. 
la centralité ${ }^{36}$ et les maux imputés au jacobinisme ${ }^{37}$, cette dynamique a trouvé dans les actes I et II de la décentralisation (Defferre, 1982 ; Raffarin, 2004) de quoi conforter des acteurs engagés dans un processus de légitimation de l'échelon local ${ }^{38}$. En s'attaquant au feuilletage administratif, les projets gouvernementaux récurrents de dévitalisation du maillon départemental au profit de régions appelées à coiffer la marqueterie territoriale ne cessent de valider ce qui, pour cesdits acteurs, constituerait le nouveau «sens de l'histoire». Reconduit à la tête de la collectivité pour un deuxième mandat, JeanYves Le Drian n'a d'ailleurs pas démenti tout l'intérêt qu'il portait à cette perspective à plus ou moins long terme : «Reconnues dans la plupart des pays européens comme le cadre institutionnel le mieux adapté à la mise en œuvre des politique publiques, les Régions ont l'avenir devant elle ${ }^{39} »$. Une manière d'accréditer l'hypothèse selon laquelle la collectivité territoriale serait désormais au cœur des «enjeux de pouvoirs ${ }^{40}$.»

On pourrait appliquer à la Région ce que Pierre Bourdieu a dit de l'État: «L'État est cette illusion bien fondée, ce lieu qui existe essentiellement parce qu'on croit qu'il existe ${ }^{41} »$. Comme l'État produirait de l'État, la Région ne devrait-elle pas, pour être pérenne, produire à son tour de la R/région ? Ce questionnement aidant, force est alors de constater que certaines décisions prises au niveau des instances locales peuvent s'avérer bien moins anodines qu'elles ne paraissent de prime abord. C'est d'ailleurs là un

36. Cf. Pierre Rosanvallon, Le modèle politique français. La société civile contre le jacobinisme, Paris, Le Seuil, 2004.

37. Michel Vovelle, Les Jacobins : de Robespierre à Chevènement, Paris, La Découverte, 1999, p. 158.

38. Maurice Agulhon, «Le centre et la périphérie», dans Pierre Nora (dir.), Les lieux de mémoire, vol. 3 : Les France, tome 1 : Conflits et partages, Paris, Gallimard, 1992, p. 825-849, p. 839-848 ; voir aussi Romain PASQUIER, La capacité politique des régions. Une comparaison France-Espagne, Rennes, Presses Universitaires de Rennes, 2004, et Alistair Cole, Beyond Devolution and Decentralisation. Building Regional Capacity in Wales and Brittany, Manchester, Manchester University Press, 2006.

39. Bretagne ensemble-Breizh a gevret, $\mathrm{n}^{\circ} 14,2010, \mathrm{p} .3$.

40. Cf. Béatrice GiBlin, «La région : enjeux de pouvoirs», Quaderni, n 59, 20052006, p. 97-108.

41. Pierre Bourdieu, Sur l'État. Cours au Collège de France (1989-1992), Paris, Raisons d'agir-Le Seuil, 2012, p. 25. 
indice de leur efficience : se faire passer pour des évidences, ce qui équivaut fréquemment, en matière de choix politiques, à la ratification du discours ambiant. Destiné aux habitants installés dans la péninsule et distribué à plus d'un million et demi d'exemplaires, Bretagne ensemble-Breizh a gevret est un semestriel dont la fonction essentielle repose sur la publicisation des actions menées par le groupe dominant de l'assemblée régionale. Il a vu le jour en 2005, un an après que la coalition de type «gauche plurielle» (Parti socialiste, Parti communiste, Parti radical de gauche, Les Verts, Union démocratique bretonne) mit fin au primat que la droite avait exercé sur le «gouvernement» local depuis les premières élections de 1986. La politique culturelle y occupe une place qui, au cours des années, n'a cessé de croître, à la fois parce que la Région est dotée en partie de cette compétence, mais aussi probablement pour d'autres raisons que nous allons essayer de cerner plus avant. "Le patrimoine culturel, fondement de notre identité et de notre attractivité», «atout rare» et "élément clé de différenciation», pouvait-on lire dans le numéro d'octobre $2009^{42}$; «Une ambition culturelle pour la Bretagne», affichait en couverture le seizième numéro (juin 2011) : argument de communication à destination intra et suprarégionale, la culture, dont la dénomination recoupe ici confusément celle de l'identité, est dorénavant tout autant un enjeu de politique publique qu'un enjeu politique tout court. La captation de la Fête de la Bretagne par la Région, qui a accentué son soutien à compter de 2009 et lui a octroyé son appellation définitive, d'origine désormais contrôlée - l'ancienne Fête de la Saint-Yves était trop religieusement connotée pour incarner cette «manifestation laïque et populaire» destinée à «promouvoir la Bretagne créative, festive et solidaire ${ }^{43}$ » -, renseigne, à bien des égards, sur sa transformation en un levier promotionnel parmi tant d'autres. L'on pourrait donc être tenté de n'y voir qu'une facette de cette stratégie de marketing territorial - un marketing qui s'accommode des autres marketings quand il n'est pas un de leurs surgeons - dans laquelle sont engagées des collectivités locales promptes à penser leur devenir en fonction de la sacro-sainte

42. Bretagne ensemble-Breizh a gevret, $\mathrm{n}^{\circ} 13,2009, \mathrm{p} .3$

43. https://web.archive.org/web/20120326185803/http://www.bretagne.fr/internet/ jcms/preprod_55620/appel-a-projets-fete-de-la-bretagne-/-gouel-breizh-2012 (16 février 2012). 
«attractivité ${ }^{44} »$. L'on cherchera prioritairement à comprendre si cet indicateur ne rend surtout pas compte de l'ajustement de la politique de la Région aux innombrables entreprises de transfiguration et d'identification qui affectent la péninsule et la condamnent à se situer ce faisant.

Car, tout autant qu'un territoire, une population ou des histoires, la Bretagne est, à ce qu'il nous semble, désormais et plus que jamais, autre chose : un intérêt né d'une somme d'intérêts particuliers plus ou moins convergents. Par intérêt, nous retiendrons l'approche qu'en propose Michel Offerlé :

«Chaque intérêt même le plus naturalisé (base locale, biologique ou professionnelle) ou le plus évident (diplôme - statut) n'est jamais que le produit d'une histoire antérieure cristallisée et mouvante à l'issue de laquelle des acteurs toujours multiples ont mis à jour, ont inventé (parfois de manière volontaire, volontariste, parfois inopinée), un groupe, des intérêts représentables ${ }^{45}$.»

Conjugaison d'une action délibérément offensive pour faire (re)connaître la spécificité bretonne - l'inauguration de la nouvelle Maison de la Bretagne à Paris en 2007 est un exemple parmi tant d'autres ${ }^{46}$ - et d'un accompagnement démultiplié en faveur des opérations culturelles qui permettent de la singulariser, la politique de la Région, qui vise à hisser toujours plus haut le pavillon régional, semble en effet répondre à une entreprise rationalisée de captation du marché identitaire.

Ce marché, ainsi qu'on l'aura constaté, se porte bien. Alimenté par des initiatives mobilisant profanes et «virtuoses de l'identité ${ }^{47}$ » à des échelles diverses, il est investi, depuis les années 1990, par

44. Christian Le BART, «Administration et pouvoir local», dans Antonin Cohen, Bernard Lacroix et Philippe Riutort (dir.), Nouveau manuel de science politique, Paris, La Découverte, 2009, p. 299-310, p. 304-306.

45. Michel Offerlé, Sociologie des groupes d'intérêt, Paris, Montchrestien, 1998, p. 64.

46. «Désormais située à Montparnasse, au cœur du Paris breton, elle assurera la présence forte, visible et permanente de la Bretagne dans la capitale», était-il signalé dans l'éditorial du septième numéro de Bretagne ensemble-Breizh a gevret, $\mathrm{n}^{\circ}$ 7, 2007, p. 3.

47. Cf. Xabier Itçaina, Les virtuoses de l'identité. Religion et politique en Pays Basque, Rennes, Presses Universitaires de Rennes, 2007. 
des associations entrepreneuriales - l'Institut de Locarn ou Produit en Bretagne pour ne citer que les plus connues - qui, en se plaçant sur le terrain de la promotion du local par le local, prônent une voie de développement endogène où la culture bretonne est considérée comme une ressource à part entière ${ }^{48}$. Il n'entre pas dans notre propos d'analyser les raisons plus ou moins formulées de l'existence de ces consortiums ni d'examiner la porosité des frontières entre agents des espaces économique, politique et intellectuel, que concrétisent les liens plus ou moins informels qu'ils entretiennent les uns avec les autres, ni de déterminer dans quelle mesure le pouvoir dont d'aucuns les dotent ne repose pas, principalement, sur les desseins qui leur sont prêtés à travers l'utilisation et la marchandisation de marqueurs culturels ${ }^{49}$. L'on remarquera en revanche que la revendication de ce «brittocentrisme», par opportunisme ou par conviction, renvoie à la réticulation d'un espace transactionnel centré sur l'identité où les concurrences sont fortes en dépit et/ou à cause des passerelles qui existent entre les «décideurs » et en dépit de la coalescence des rôles - celle qui conduit certains agents à arguer de leurs compétences pour être à la fois ses observateurs et ses prescripteurs. Il n'aura peutêtre pas échappé aux lecteurs du récent Livre blanc de la Bretagne qu'il a paru sous les auspices de Bretagne Prospective. Né des réflexions menées par quelques dizaines de collaborateurs provenant de divers horizons dans le cadre de ce laboratoire d'idées que préside Jean Ollivro, professeur de géographie à l'Université Rennes 2, l'ouvrage est conçu comme un programme pour une «Bretagne [qui] semble parfois menacée de disparition», ainsi que le souligne, d'entrée de jeu, la première ligne d'une introduction intitulée au demeurant $"$ Créer un pays différent ${ }^{50} »$. La culture y très présente au motif qu'elle serait «le levier décisif d'une nouvelle forme de

48. Ronan Le CoAdic, L'Identité bretonne, op. cit., p. 282-284 ; Yann Fournis, Les régionalismes en Bretagne. La région et l'État (1950-2000), op. cit., p. 223-231.

49. Anne Guillou et Christian PAPINOT, La valeur de l'image : l'usage de la symbolique bretonne dans la promotion des produits industriels et artisanaux dans le Finistère. Étude sociologique, Brest, Université de Bretagne Occidentale, 1998, p. 18.

50. Bretagne Prospective, Le livre blanc de la Bretagne. Enjeux et Perspectives, Nantes, Éditions du Temps, 2008, p. 7. 
construction ${ }^{51} »$. Les « préconisations », quant à elles, concernent aussi bien «la connaissance de la matière de Bretagne», envisagée comme «un socle qui permet d'agir pour le futur et d'engager des projets adaptés à l'identité de notre pays ${ }^{52}$ », que la promotion de la langue bretonne dans les entreprises (sur le modèle catalan dûment pris pour exemple). Partie prenante dans un marché identitaire qui ne cesse de sécréter une compétition entre des acteurs certes différemment pourvus en capitaux (économiques, politiques, symboliques) mais tous soucieux de faire valoir ce que doit être l'intérêt de la Bretagne pour faire avancer aussi leur propre intérêt ${ }^{53}$, le Conseil régional est donc «sommé» de se positionner au risque, sinon, de se laisser distancer sur cette question. Ce positionnement repose à nos yeux sur la fabrication d'un «consensus ambigu ${ }^{54} »$.

La Région semble être en effet aujourd'hui l'échelon de l'avenir. Le contexte s'y prête en raison d'une remise en cause progressive de l'ordre stato-national, dont la France aurait été une de ses incarnations les plus abouties ${ }^{55}$, et de l'avènement de cette «Europe des régions », un slogan dont les élites locales usent abondamment dans leurs discours pour justifier de leur ancrage et de leur disponibilité à exercer des fonctions, alors même que certaines analyses attestent combien la redistribution des pouvoirs à l'intérieur de l'Union européenne est bien plus complexe que ces discours ne l'affirment ${ }^{56}$. En Bretagne, les années Le Drian apparaissent, à bien des égards, comme un miroir grossissant de ces mutations politico-territoriales pour des raisons qui tiennent autant à une stratégie politique - et, peut-être, à des convictions personnelles - qu'à la généalogie de l'institution régionale. Un appareil bureaucratique balbutiant tra-

51. Ibid., p. 13.

52. Ibid., p. 8-9.

53. Une hypothèse que seule une étude ethnographique fouillée des réseaux et des relations irriguant cet espace transactionnel permettrait ou non de valider.

54. En référence au livre de Mark Kesselman qui, dans un tout autre contexte, analysait les modes de fonctionnement du gouvernement local.

55. Jean-François CHANET, «Terroirs et pays : mort et transfiguration ?», Vingtième Siècle. Revue d'histoire, $\mathrm{n}^{\circ}$ 69, 2001, p. 61-81.

56. Patrick Le Galès et Christian LeQUeSNe, «Introduction », dans Patrick Le Galès et Christian Lequesne (dir.), Les paradoxes des régions en Europe, Paris, La Découverte, 1997, p. 9-15. 
vaillant de conserve avec les notables après les «années Célib ${ }^{57}$ » qui en avaient facilité les rapprochements, une propension des élus à utiliser leurs réseaux nationaux pour faire avancer leurs dossiers locaux et ce, au moins, depuis le principat du gaulliste Yvon Bourges à la tête de la collectivité (1986-1998), un échafaudage identitaire qui ne demandait qu'à s'élever après que Josselin de Rohan (RPR) eut confié au centriste Jean-Yves Cozan une vice-présidence «en charge de l'identité bretonne et de la culture» pendant son mandat (1998-2004) : autant d'éléments matriciels qui auront ouvert la voie à l'affirmation possible d'un leadership procédant directement de l'instance représentative et délibérative.

$\mathrm{Au}$ surplus, dans une Bretagne qui, à la différence de la plupart des régions dont «le propre (...) est de n'avoir ni définition ni contour ${ }^{58} »$, peut incarner le contraire, l'histoire et ses usages sont susceptibles d'être aisément mis à contribution pour attester la continuité pluriséculaire d'un substrat identitaire et, partant, l'inéluctabilité d'un retour en grâce de son supposé débouché statoterritorial ${ }^{59}$. L'alternance partisane de 2004 ne se sera d'ailleurs traduite que par l'approfondissement d'une politique culturo-identitaire demeurée jusqu'alors plus ou moins en filigrane : une continuité, pardelà l'apparente discontinuité électorale, qui rappelle aussi combien toute institution, surtout lorsqu'elle est récente - ce qui est toujours le cas du Conseil régional -, impose à ses agents de se conformer à ses exigences et, entre autres, à un passé qu'ils peuvent utiliser à leur tour pour défendre leurs actions et négocier leurs intérêts ${ }^{60}$. L'on en voudra pour preuve, par exemple, le choix du logo dont s'est dotée la collectivité en 2005 et la présentation qui l'accompagne : «Son nouveau logo a gardé les points forts de l'ancien : la forme

57. Dans les années 1950 et 1960, le Comité d'étude et de liaison des intérêts bretons (Célib) a été à l'origine d'une expérience pionnière par son ampleur et sa capacité à imposer à l'administration centrale la prise en compte des besoins régionaux.

58. Marie-Claude SMOUTS, «La région comme nouvelle communauté imaginaire?», dans Patrick Le Galès et Christian Lequesne (dir.), Les paradoxes des régions en Europe, op. cit., p. 37-45.

59. François Hartog et Jacques ReVEL, «Note de conjoncture historiographique», dans François Hartog et Jacques Revel (dir.), Les usages politiques du passé, Paris, Éditions de l'EHESS, 2001, p. 13-24, p. 19.

60. Cf. Jacques Lagroye et Michel Offerlé (dir.), Sociologie de l'institution, Paris, Belin, 2010. 
carrée, un intitulé clair (Région Bretagne), des couleurs (le bleu et le vert) symbolisant à la fois l'Argoat (la terre) et l'Armor (la mer). Mais il a évolué vers un sigle au design à la fois plus moderne (couleurs et typographie) et plus porteur de l'identité bretonne. Une hermine très stylisée reprend la forme géographique de la Bretagne, évoquant ainsi à la fois les racines et le dynamisme d'une Bretagne en mouvement ${ }^{61} \gg$. L'on notera au passage que la réminiscence graphique de l'hermine ducale, cette inflexion supplémentaire apportée à la formalisation d'un discours légitimiste qui ne cesse de ratifier l'idée d'une préemption de l'histoire sur le présent et l'avenir - le duché du Moyen Âge avec Nantes comme capitale en tant que préfiguration d'un possible remaniement territorial -, ne suscita guère de commentaires au moment où elle vit le jour (parce qu'elle paraissait anodine ? parce que les conditions de possibilité de son existence étaient enfin réalisées ?).

Porté par le Conseil régional, ce travail de mobilisation symbolique et de coproduction identitaire suppose un consentement $a$ minima que la réalité du pouvoir local facilite aisément après qu'il semble être devenu, aux yeux de nombreux élus, le pilier régénérateur de la démocratie représentative ${ }^{62}$. Consensus ambigu, avons-nous donc écrit. Il repose, au nom de l'existence d'un bien commun, sur la dépolitisation d'une politique culturo-identitaire conçue comme une politique non partisane ${ }^{63}$. Il se fabrique en partie grâce à la neutralisation des affiliations idéologiques par objectivation d'éléments supposément fédérateurs (signes, lieux, personnages célébrés, etc.) comme en témoigne, par exemple, le discours prononcé le 15 décembre 2011 par Jean-Michel Le Boulanger, vice-président du Conseil régional «en charge de la culture et des politiques culturelles » à l'occasion des trente ans de

61. http://emblemes.free.fr/site/index.php?option=com_content\&view=article\&id $=427: \log$ os-du-conseil-regional-de-bretagne $\&$ catid $=52:$ bretagne $\&$ Itemid $=102$ (16 février 2012).

62. Michel Koebel, Le pouvoir local ou la démocratie improbable, Bellecombe-enBauges, Éditions du Croquant, 2006, p. 9.

63. On notera, par exemple, que dans la page de Bretagne ensemble-Breizh a gevret consacrée à l'expression des groupes politiques représentés au Conseil régional, l'opposition de droite et du centre signe sous le titre «Breizh da Zont - Bretagne à Venir» depuis le numéro 14 (octobre 2010), soit après le dernier scrutin régional, alors qu'elle signait jusque-là sous le titre «Groupe UMP». 
la disparition de l'auteur du Cheval couché : «Rendre hommage à Xavier Grall, ici, dans l'hémicycle du Conseil régional de Bretagne, c'est dire collectivement ce que la Bretagne doit à ceux qui ont porté son chant quand les temps étaient difficiles encore. L'identité souriante et apaisée d'aujourd'hui a été précédée de combats et de cris. La place de la langue et de la culture, la place d'une évidence, de ces prénoms que l'on choisit et de ces Gwenn ha Du qui flottent librement, tranquillement, cette place, elle a été gagnée, pas à pas. Et les poèmes de Xavier Grall y ont un rôle majeur ${ }^{64}{ }^{\prime}$.

Ce consensus ambigu et fonctionnel, qui écrête les oppositions en promouvant des représentations à vocation conciliatrice, ne saurait occulter toutefois les dimensions proprement idéologiques d'une entreprise d'investigation/investissement de l'identité. Lancée en grandes pompes par la Région en 2011, la Marque Bretagne générée dans le but d' "élargir, rajeunir et dynamiser l'image de la Bretagne afin d'attirer de nouvelles clientèles et de développer la notoriété de la région à l'international ${ }^{65} »$ ressortit évidemment à ce marketing territorial que nous avons succinctement évoqué. Portée sur les fonts baptismaux de la communication institutionnelle, suite à une enquête auprès de quelque $« 5000$ Bretons ${ }^{66} »$ qui devait aboutir à la détermination d'un panorama de la péninsule, elle est revêtue d'une double mission : "symbolise[r] le territoire, lui confére[r] une valeur ajoutée, un "supplément d'âme" qui renforce sa personnalité et lui apporte ce "sens" tant recherché par les clients aujourd'hui, tant dans le domaine économique et universitaire que touristique, culturel ou sportif»; "participe[r] (...) au renforcement de la fierté d'appartenance, à la mobilisation et à la fédération de ses acteurs les plus entreprenants ${ }^{67} \gg$. Abondante, la documentation qui renseigne l'internaute sur les objectifs de la Marque fait la part belle à un compendium électronique de près de neuf cents pages qui décline les portraits «physique» et "psychologique» de la Bretagne avant d'en proposer une synthèse valorisant, ici aussi, «un modèle

64. https://www.europe.bzh/jcms/preprod_132150/xavier-grall (16 février 2012). C'est nous qui soulignons.

65. Bretagne ensemble-Breizh a gevret, $\mathrm{n}^{\circ}$ 15, 2011, p. 24.

66. Ibid.

67. https://web.archive.org/web/20120210231653/http://www.marque-bretagne.fr/ code-de-marque/introduction (16 février 2012). 
breton ${ }^{68}$ ». Le curieux y trouve donc matière à lecture : «Rapport avec l'histoire : Une singulière et "très longue histoire" régionale, mais une mémoire à "trous", notamment une histoire régionale occultée au profit de l'histoire de France, non enseignée et donc méconnue de nombreux Bretons eux-mêmes. Une Bretagne au destin mouvementé et souvent contrarié, fait de nombreuses périodes de lutte et de résistance, indépendante durant un peu moins de 7 siècles, à l'histoire française de moins de 5 siècles, ayant forgé une force et un "sentiment breton" collectifs extra-ordinaires où la Bretagne historique reste omniprésente. En héritage, un caractère, des valeurs particulièrement solides et un sens aigu du collectif, mais aussi, en arrière-fond du tempérament, l'empreinte sombre des souffrances éprouvées ${ }^{69} 》 ;$ « Caractère \& comportements : (...) Tempérament : un esprit breton forgé par les éléments ; attachement prononcé, viscéral et passionnel des Bretons à leur territoire, modérés, égalitaires ; goût de l'indépendance et une certaine complaisance dans un état de permanente revendication face à l'État, fierté, volonté collective de solidarité et "de s'en sortir tout seuls" ; goût séculaire des Bretons pour la compétition, esprit combatif (...). Relations avec les autres : pudeur, modestie et fierté, sérieux, grande sensibilité allant parfois jusqu'à une certaine susceptibilité, simplicité, authenticité et grande spontanéité dans les relations, pour le pire comme pour le meilleur... ${ }^{70} »$.

L'étude d'une source risquant d'entraîner immanquablement son effet en induisant un regard biaisé parce qu'univoque, nous ferons appel à des traces supplémentaires qui sanctionnent l'établissement d'un discours d'édification censé épouser la "pulsion régionale ${ }^{71}$ » et donc apte à la traduire. Devenue une icône pour certains (son nom a été donné à des institutions publiques et privées), Anjela Duval (1905-1981) a ceci de possiblement fédérateur qu'elle combine le triple statut de femme, de paysanne et d'écrivain de langue bretonne, à la croisée d'un art savant (la poésie écrite) et d'une extraction

68. https://fr.calameo.com/read/00000131512571b3b65ad (16 février 2002), page 861.

69. Ibid., p. 805.

70. Ibid., p. 808.

71. Emmanuel Le Roy Ladurie, Histoire de France des régions. La périphérie française des origines à nos jours, Paris, Le Seuil, 2005 [2001], p. XI. 
populaire. Inaugurée au Vieux-Marché le 6 novembre 2011, la sculpture offerte à sa mémoire fut l'occasion pour le vice-président du Conseil régional, qui avait soutenu l'initiative, de prononcer un éloge dont les premiers mots furent : «Il ne faut jamais renier les siens, il ne faut jamais oublier ce que l'on doit à ceux qui nous ont précédés et il est bon que les Bretons nombreux se soient mobilisés pour cette statue ${ }^{72}$.»

Mis bout à bout, ces mots fabriquent un puzzle d'où il ressort, par-delà des expressions qui empruntent à une taxinomie héritée de la psychologie des peuples et des études consacrées aux personnalités ethniques - l'ouvrage électronique de la Marque Bretagne en est truffé -, des catégories à la mode dans le champ non structuré des sciences sociales de la fin du dix-neuvième siècle ${ }^{73}$, une équation associant asymétriquement universalité et enracinement. Car, derrière l'universalité, c'est d'abord aux racines et à un cortège d'opérations visant à les maintenir ou à les faire exister qu'il est fait plus ou moins expressément référence. Pour ceux qui en sont les vecteurs, cette autochtonisation qui ne dit pas son nom ${ }^{74}$, dont les accents ressemblent à s'y méprendre à certaines inflexions barrésiennes ou lavissiennes ${ }^{75}$, et qui plaide en faveur d'un réexamen des relations entre culture d'en haut et culture d'en bas - pour reprendre le titre de l'ouvrage de Laurence W. Levine ${ }^{76}$-, ne saurait d'ailleurs demeurer sans incidence sur le devenir d'une société régionale à régionaliser.

72. https://abp.bzh/anjela-duval-23771 (16 février 2012). Prononcé lui aussi par le vice-président du Conseil régional «en charge de la culture et des politiques culturelles», l'hommage en l'honneur de Xavier Grall se concluait par ses mots : "Xavier Grall, "visage terrible et magnifique, traversé par un éclair” (Yvon Le Men), est décédé il y a trente ans. La Bretagne lui doit beaucoup. Il ne faut jamais renier les siens», https://www.europe.bzh/jcms/preprod_132150/xaviergrall (16 février 2012).

73. Laurent MucChielli, La découverte du social. Naissance de la sociologie en France (1870-1914), Paris, La Découverte, 1998.

74. Cf. Marcel Detienne, Comment être autochtone. Du pur Athénien au Français raciné, Paris, Le Seuil, 2003.

75. Marcel Detienne, L'identité nationale, une énigme, Paris, Gallimard, 2010, p. 49-70.

76. Laurence W. Levine, Culture d'en haut, culture d'en bas. L'émergence des hiérarchies culturelles aux États-Unis, Paris, La Découverte, 2010 [1988]. 
Au nom de cette subjectivation de la Bretagne qui supposerait d'en passer aussi par là.

Grand orchestrateur d'une politique culturo-identitaire, le Conseil régional s'est finalement imposé comme un des acteurs majeurs - pour ne pas dire l'acteur majeur - dans la mobilisation du schème identitaire. Son entreprise polymorphe confirme, ce faisant, les analyses d'un Pierre Bourdieu qui affirmait, il y a plus de trente ans, que la région était ce laboratoire où les tenants de l'identité luttaient surtout discursivement pour conquérir le «monopole de [s]a définition légitime ${ }^{77}$ ». Auréolée de son incarnation en tant que corps politique et, donc, de sa visibilité dans l'espace public - que consacre d'ailleurs le magazine de la collectivité, cette "propagande masquée ${ }^{78} »-$, la Région peut empoigner d'autant plus aisément cet objet que le consensus politique ambigu sur lequel elle s'appuie paraît rejoindre un consensus identitaire que distillent, chacun à sa manière, dans un espace à la fois éminemment polycentrique et imbriqué, observateurs et/ou prescripteurs de la question. L'on fera d'ailleurs remarquer que la force apparente de ce double consensus est telle que, tandis que le «roman national» est déprécié, le «roman régional» est, quant à lui, sinon valorisé, à tout le moins très minoritairement soumis à la critique... alors même que ses fondements théoriques et sa mise en pratique n'échappent pas, comme par duplication, et dans une forme euphémisée, à ce modèle tertio-républicain de nationalisation des masses qui constitue son utile repoussoir. Un mouvement symétrique qui en dit finalement long sur certaines logiques présidant à la (re)localisation de l'identité et sur l'intérêt que des partis politiques peuvent y trouver. Aussi, nous interrogerons-nous, pour finir, sur l'utilisation que les socialistes, aux commandes de la collectivité depuis maintenant huit ans, semblent en faire, tant les équipes de Jean-Yves Le Drian ont alimenté le «kit» identitaire ${ }^{79}$ en marqueurs de toutes sortes. Héritiers de la tradition d'une gauche

77. Pierre BourdieU, «L'identité et la représentation. Éléments pour une réflexion critique sur l'idée de région», Actes de la recherche en sciences sociales, $\mathrm{n}^{\circ} 35$, 1980, p. 63-72, p. 63.

78. Michel Koebel, Le pouvoir local ou la démocratie improbable, p. 64.

79. Anne-Marie Thiesse rappelle que le terme même de kit appliqué à l'identité est dû à l'ethnologue Orvar Löfgren; La création des identités nationales. Europe, XVIII -XXe siècle, Paris, Gallimard, 2001 [1999], p. 13. 
non communiste adepte de La France au pluriel $^{80}$ qui disposa, en Bretagne, de ses ténors rompus, dès les années 1970, à un discours régionaliste et décentralisateur ${ }^{81}$, les représentants actuels du Parti socialiste ne feraient qu'entériner un état de fait. L'orientation donnée à la politique culturo-identitaire mérite que nous allions au-delà de cette assertion centrée sur la seule filiation idéologique et que nous formulions quelques hypothèses complémentaires. Dans un parti où la référence ouvriériste et, plus globalement, la question sociale ne servent plus à agréger des militants et à personnifier la transformation possible du pays ${ }^{82}$, place est offerte à des thèmes beaucoup plus transversaux dont la capacité à solidariser un appareil militant et un électorat qui, eux aussi, ont sociologiquement beaucoup évolué ${ }^{83}$, est désormais incommensurable. Perçue comme une ressource à la fois individualisable et collective à destination d'une société atomisée qui se pense moins à travers les catégories sociales qu'à travers les affinités électives, l'identité, surtout lorsqu'elle se matérialise à travers la culture, se mue immanquablement en un objet à ne pas négliger. Au surplus, dans une démocratie qui, depuis une décennie, a fait de la proximité le gage de son ressourcement et fabriqué, sous son égide, de nouvelles légitimités ${ }^{84}$, l'identité tombe à point nommé : elle dispose en effet d'une efficacité d'autant plus grande qu'elle n'implique pas de dotations budgétaires conséquentes et joue pleinement sur la prime accordée au local. Si l'on ajoute que, depuis l'élection présidentielle de 2007, le «modèle picto-charentais» a été érigé par certains caciques du Parti socialiste en un laboratoire de la conquête du pouvoir central, sous couvert de l'expérience/ expertise du terrain et du rapprochement avec les citoyens, et

80. Titre de l'ouvrage paru sous la direction de François Mitterrand en 1981 aux éditions Entente.

81. Barbara LOYER, «Bretagne», op. cit., p. 277.

82. Rémi Lefebvre et Frédéric SAWICKI, La société des socialistes. Le PS aujourd'hui, Bellecombe-en-Bauges, Éditions du Croquant, 2006, p. 222-232.

83. Avec la montée en puissance des classes moyennes et moyennes supérieures à fort capital intellectuel ; cf. Rémi LEFEBVRE et Frédéric SAWICKI, La société des socialistes. Le PS aujourd'hui, Bellecombe-en-Bauges, op. cit.

84. Cf. Christian Le BART et Rémi LefebvRE, «Introduction : Une nouvelle grandeur politique ?», dans Christian Le Bart et Rémi Lefebvre, La proximité en politique. Usages, rhétoriques, pratiques, Rennes, Presses Universitaires de Rennes, 2005, p. 11-30. 
si l'on tient compte des stratégies personnelles de certains de ses élus régionaux ${ }^{85}$, l'on gagera que la politique menée par JeanYves Le Drian, cet «entrepreneur de proximité ${ }^{86}$ », en s'adossant à l'identité, cette idée qui se donne à voir et à s'exprimer, répond aussi vraisemblablement à un impératif encore plus politique qu'il n'y paraît : «périphériser» davantage la périphérie afin de gagner le centre en arguant de sa propre consécration par la périphérie ${ }^{87}$. En d'autres termes, hypostasier la région ${ }^{88}$ - et la Région - pour exister un peu plus à l'échelle de la nation, quitte à exhumer la figure du grand feudataire ${ }^{89}$, cette figure d'une modernité que d'aucuns pourraient qualifier de régressive.

\section{$* * *$}

C'est une petite musique de l'identité, un discours éclaté (et rodé) qui se doit de fonctionner par imprégnation et infuser. Émanation de producteurs/prescripteurs qui émargent à des sphères différentes, elle s'apparente, à bien des égards, à celle qui participa à la construction des nations européennes au cours du dix-neuvième siècle ${ }^{90}$. Un siècle où l'on vit des élites rendre cohérentes des com-

85. On se reportera, par exemple, au portrait de Jean-Yves Le Drian paru dans Le Télégramme du 4 avril 2004.

86. Ibid., p. 18.

87. Cet article a été terminé en mars 2012, avant que échéances électorales d'avrilmai n'aient lieu et que le premier gouvernement d'un président de la République qui pourrait être François Hollande ne fasse - ou non - une place à Jean-Yves Le Drian.

88. Un processus qui ne concerne pas seulement les agents politiques : «À l'orée du XXI ${ }^{\mathrm{e}}$ siècle, le contexte européen offre ainsi un cadre favorable à la consolidation, à l'épanouissement d'une Bretagne maîtresse de son propre destin, au-delà des atteintes et des altérations portées par l'État français»; «Plus que jamais, cette vieille province, si passionnément bretonne et si éminemment française, nous offre une belle leçon politique. Elle est un laboratoire d'expériences susceptibles d'articuler harmonieusement un continent (l'Europe), une nation (la France) et une région, farouchement, pleinement et définitivement... bretonne», Joël CoRnette, Histoire de la Bretagne et des Bretons, tome 2 : Des Lumières au XXIe siècle, op. cit., p. 599 et 601.

89. Christian LE BART, «Administration et pouvoir local», op. cit., p. 310.

90. Cf. Eric Hobsbawm, Nations et nationalisme depuis 1780. Programme, mythe, réalité, Paris, Gallimard, 2001 [1990]. 
munautés imaginaires ${ }^{91}$ en excipant de ces nouvelles hiérarchies culturelles appelées à devenir plus ou moins arbitrairement, grâce à l'intérêt que leur portèrent des entrepreneurs politiques, des normes de référence à destination des populations ${ }^{92}$. L'ordre régional tel qu'il se constitue et s'organise actuellement en Bretagne se donne ainsi à lire mais aussi à voir. L'on se souviendra peut-être que le candidat Le Drian fit campagne en 2004 en arborant un ciré jaune Cotten. L'on notera qu'en 2010, pour sa réélection, le président de la Région porta une chemise noire et une cravate jaune criblée d'hermines. Signes faussement anecdotiques, car utilisés à dessein, ils pourraient être définis, à partir de la lecture extensive des Deux Corps $d u$ Roi ${ }^{93}$ proposée par Pierre Bourdieu, comme les indices dont se pare «le chef de maison [qui] est en quelque sorte l'incarnation provisoire de cette unité transcendante qu'est la maison ${ }^{94} \gg$. Une politique d'affichage qui serait, en d'autres termes, une façon toute personnelle d'incorporer une Bretagne transfigurée.

91. Benedict ANDERSON, L'imaginaire national. Réflexions sur l'origine et l'essor du nationalisme, Paris, La Découverte, 2002 [1983], p. 93-118.

92. Ernest Gellner, Nations et nationalisme, Paris, Payot, 1989 [1983], p. 84-88.

93. Ernst Kantorowicz, 1989 [1957], Les Deux Corps du Roi. Essai sur la théologie politique au Moyen Âge, Paris, Gallimard, 1989 [1957].

94. Pierre Bourdieu, Sur l'État. Cours au Collège de France (1989-1992), op. cit., p. 389. 